\title{
Linx
}

Revue des linguistes de l'université Paris X Nanterre

$62-63 \mid 2010$

Entre syntaxe et discours. Éclairages

épistémologiques et descriptions linguistiques

\section{Indexicaux, discours et mémoire discursive : ce que les premiers révèlent du second et de la troisième}

\section{Francis Cornish}

\section{OpenEdition}

\section{Journals}

Édition électronique

URL : http://journals.openedition.org/linx/1377

DOI : $10.4000 /$ linx.1377

ISSN : 2118-9692

\section{Éditeur}

Presses universitaires de Paris Nanterre

Édition imprimée

Date de publication : 1 juillet 2010

Pagination : 111-133

ISSN : 0246-8743

\section{Référence électronique}

Francis Cornish, «Indexicaux, discours et mémoire discursive : ce que les premiers révèlent du second et de la troisième », Linx [En ligne], 62-63 | 2010, mis en ligne le 04 décembre 2013, consulté le 05 mai 2019. URL : http://journals.openedition.org/linx/1377 ; DOI : 10.4000/linx.1377 


\title{
Indexicaux, discours et mémoire discursive : ce que les premiers révèlent du second et de la troisième
}

\author{
Francis Cornish \\ Université de Toulouse-Le-Mirail et CNRS CLLE-ERSS, UMR 5623 \\ cornish@univ-tlse2.fr
}

\author{
Introduction \\ D’après Arie Verhagen (2005, p. 22), ${ }^{1}$ \\ les expressions langagières sont en premier lieu des indices («cues ») pour faire des \\ inférences, et la compréhension ne consiste pas essentiellement dans le décodage du \\ contenu exact des expressions [dans un texte quelconque], mais dans la création \\ d'inférences qui conduisent à des interventions (cognitives, conversationnelles ou \\ comportementales) subséquentes qui s'avéreront adéquates.
}

Ce principe va encadrer le propos de cet article. Il s'agira de voir comment le fonctionnement de certains types d'indexicaux (pronoms de $3^{\mathrm{ème}}$ personne, SN définis et démonstratifs) sert dans leur contexte énonciatif à révéler et à alimenter l'état des représentations discursives en cours d'élaboration au sein du discours et de la mémoire discursive (MD) (le modèle mental de ce discours élaboré dans l'esprit de chaque interlocuteur, ou scripteur/lecteur).

${ }^{1}$ Je traduis. 
Je commencerai par poser les bases théoriques pour les analyses à venir, en esquissant le cadre dans lequel je travaille actuellement (la triple distinction entre texte, contexte et discours, et leurs rôles respectifs dans la communication) (section 1). Ensuite je caractériserai les procédures référentielles de deixis et d'anaphore en fonction d'une conception «scalaire » des relations entre ces deux moyens de coordination du foyer d'attention des interlocuteurs, en me concentrant surtout sur ce qu'Ehlich (1982) appelle «l'anadeixis » - une forme de référence indexicale qui combine les procédures déictique et anaphorique à des degrés divers (section 2).

Dans la partie centrale du travail (section 3), j'appliquerai le cadre et les outils analytiques esquissés dans les sections précédentes à l'analyse de quatre extraits d'enregistrements de conversations guidées ou libres tirées du projet sur la Phonologie du Français Contemporain (PFC). Ces extraits comportent plusieurs types d'expressions indexicales, qui révèlent et même contribuent à l'existence ainsi qu'au statut discursivo-cognitif de certaines représentations mentales des référents concernés au moment de l'occurrence. Cette discussion nous permettra de prendre conscience des écarts qui peuvent exister entre ce que j’appelle le texte et le discours (voir la distinction analogue que fait Johnsen (à paraitre, $\mathbb{S} 1.3$ ) à la suite de Berrendonner entre « la combinatoire des éléments régis » et « celle des actions communicatives »), et de prendre toute la mesure du rôle du contexte à l'œuvre dans la construction d'une interprétation située d'un acte d'énonciation quelconque. Enfin, dans la Conclusion, je ferai le point sur les types de modulation que des renvois indexicaux peuvent effectuer sur l'état de la MD au moment de ces renvois, et indiquerai comment ces représentations peuvent être formulées et alimentées.

\section{Préliminaires conceptuels et terminologiques}

Commençons donc par faire quelques distinctions terminologiques et conceptuelles qui vont être indispensables pour ce qui suit. D'abord, la triple distinction texte/ contexte/discours peut se caractériser comme suit (voir aussi Widdowson, 2004, ch.1) :

Tableau 1 : Rôles du texte, du contexte et du discours dans l'acte d'énonciation

\begin{tabular}{|c|c|c|}
\hline Texte & Contexte & Discours \\
\hline $\begin{array}{l}\text { La séquence connexe de signes } \\
\text { verbaux et de signaux non- } \\
\text { verbaux en fonction de laquelle } \\
\text { le discours est co-construit par } \\
\text { les participants dans l'acte } \\
\text { de communication. }\end{array}$ & $\begin{array}{l}\text { Le contexte (le domaine de référence } \\
\text { d'un texte donné, le co-texte, le } \\
\text { discours déjà construit en amont, le } \\
\text { genre d'événement langagier en } \\
\text { déroulement, le cadre socio-culturel } \\
\text { supposé par le texte, les relations } \\
\text { interlocutives existant entre les interactants } \\
\text { à chaque moment de l'échange, et la } \\
\text { situation d'énonciation particulière à } \\
\text { l'œuvre) est assujetti à un processus } \\
\text { continu de construction et de révision } \\
\text { au fur et à mesure que le discours se } \\
\text { déroule. C'est à travers l'invocation } \\
\text { d'un contexte approprié que l'auditeur } \\
\text { ou le lecteur peut convertir en discours } \\
\text { la séquence connexe d'indices textuels } \\
\text { qu'est le texte. }\end{array}$ & $\begin{array}{l}\text { Le produit de la séquence } \\
\text { hiérarchisée et contextuellement } \\
\text { située d'actes énonciatifs, } \\
\text { indexicaux, propositionnels, et } \\
\text { illocutoires effectués dans la } \\
\text { poursuite d'un but communicatif } \\
\text { quelconque, et intégrés dans } \\
\text { un contexte donné. }\end{array}$ \\
\hline
\end{tabular}


Selon moi, le texte (voir la colonne de gauche du tableau 1) est la trace d'au moins un acte d'énonciation (qu'il soit réalisé sous la forme d'une trace verbale, linguistique, ou bien non-verbale - qui, elle, peut être gestuelle, sensori-perceptuelle ou prosodique). Parmi les indices ou signaux non-verbaux se trouvent la direction du regard, les clins d'œil, le froncement des sourcils, et les gestes de pointage de divers types; et dans la forme écrite, ils comprennent le soulignement, les italiques, les caractères gras, la ponctuation, la division en paragraphes et la typo-disposition en général. La notion de texte, donc, renvoie aux séquences connexes de signes et de signaux, dans leur signification conventionnelle, telles qu'elles sont produites par l'énonciateur et (dans le cadre des interactions parlées familières) également par le coénonciateur. Certains des signes et signaux ainsi produits pointent vers des manières possibles d'ancrer le discours à construire dans un contexte particulier, en termes cognitifs (voir aussi Langacker, 2002a, 2002b). Ces signaux correspondent à ce que Gumperz (1992a, p. 234) dénomme «indices de contextualisation » (voir aussi Auer, 1992, de même que Gumperz, 1992b).

Le contexte pertinent pour un acte d'énonciation quelconque est un composé du co-texte environnant, du domaine de référence à l'œuvre, du genre d'événement langagier qui est en déroulement, de la situation d'énonciation particulière, du discours déjà construit en amont, des relations interlocutives existant entre les interactants à chaque moment de l'échange, et du cadre socio-culturel supposé par le texte (voir la colonne du milieu du tableau 1). Or, ce contexte ne pré-existe pas au discours de façon objective ou externe, mais est (re-)créé en permanence à chaque instant de la communication - il est donc en développement constant : le discours construit à partir du texte en dépend, en même temps qu'il le change au fur et à mesure qu'il est créé en temps réel. Le contexte invoqué servira à sélectionner la signification appropriée des lexèmes, l'ajustera afin de la rendre compatible avec le discours déjà construit, et fonctionnera en général pour désambiguïser les multiples interprétations possibles de certains des segments textuels. Il permettra également de préciser les références elliptiques ou autrement indéterminées qu'il pourrait y avoir dans le co-texte, et d'étoffer des allusions faites dans le texte à certains aspects de la connaissance du monde. De plus, il aidera le destinataire à déterminer la force illocutoire de chaque proposition arrivante ${ }^{2}$.

Quant au discours, il renvoie au produit hiérarchiquement structuré, mentalement représenté, de la séquence d'actes énonciatifs, propositionnels, illocutoires et indexicaux que les participants effectuent au fur et à mesure que la communication se déroule. L'organisation hiérarchique du discours reflète le fait que les unités discursives sont articulées en unités d'arrière-plan, d'ancrage modal et référentiel, et en unités de premier plan, de nature à faire avancer la communication. De telles séquences ont pour raison d'être la réalisation d'un but communicatif local et/ou global quelconque (voir Parisi et Castelfranchi, 1977). Le discours dépend à l'évidence à la fois du texte et du contexte. C'est le discours construit en fonction du texte et d'un contexte pertinent qui est capable d'être stocké par la suite dans la mémoire à long terme, en attendant

${ }^{2}$ Voir aussi Cornish (2009) à ce sujet. 
une récupération ultérieure possible ${ }^{3}$. Par contre, la trace textuelle de l'événement communicatif est éphémère (du moins à l'oral), car elle disparait de la mémoire à court terme dès la construction du discours - ou peu de temps après (cf. Jarvella 1979).

Le point crucial dans cette distinction est que le discours est une (re)construction mentale, et donc hautement probabiliste : du point de vue de l'allocutaire, il ne s'agit nullement d'un simple décodage du texte afin d'arriver au message complet voulu par l'énonciateur (voir la citation de Verhagen, 2005 donnée en exergue). Le discours, donc, est un construit à la fois hiérarchisé et défaisable (la construction provisoire et révisable d'une interprétation située), alors que le texte est essentiellement linéaire cependant, dans la forme orale, des phénomènes paralinguistiques, non-verbaux peuvent co-apparaittre simultanément avec le flux des signes verbaux et d'autres types de signaux non-verbaux. Le 'sens' ne réside pas entièrement «dans » le texte, il doit être construit par l'allocutaire (et l'énonciateur!) via le texte et un contexte approprié. De toute manière, le texte est souvent, sinon toujours, à la fois incomplet et sousspécifié par rapport au discours qui peut être construit à l'aide du contexte. Il sousdétermine donc de façon importante ce discours (l'interprétation suivie et située en contexte) ${ }^{4}$. Texte, contexte et discours sont donc interdépendants, interactifs et s'entre-définissent.

\section{Deixis, « anadeixis " et anaphore : une conception « scalaire »}

\subsection{Définitions}

La deixis et l'anaphore sont des moyens de gestion de la coordination de l'attention des participants du discours. L'utilisateur exploite ces procédures discursivo-référentielles pour construire, modifier et avoir accès au contenu de modèles du discours en déroulement, qui sont représentés mentalement par chacun des participants au discours (cf. le terme de "mémoire discursive » proposé par Marie-José [Reichler-] Béguelin, 1988 et Alain Berrendonner, 1983). Ces deux procédures opèrent en fonction du principe selon lequel le discours serait conçu en rapport avec les conditions de réception du texte concerné chez l'allocutaire, telles qu'elles sont supposées par l'énonciateur - à savoir, le principe d'intersubjectivité (cf. entre autres Benveniste, 1974, p. 82).

La deixis sert prototypiquement à orienter le foyer d'attention de l'allocutaire vers un nouvel objet de discours (ou vers un nouvel aspect d'un objet de discours déjà existant au moment de la prise de parole) qui est à construire mentalement, par défaut à partir de la situation d'énonciation - dont le centre (l'« origo » chez Bühler, 2009[1934]) est le bic et nunc de l'activité verbale et non-verbale du locuteur (voir aussi Diessel, 2006, p. 470). La deixis, dans cette conception, implique l'exploitation du contexte d'énonciation (le fond ("ground») déictique dans la terminologie de Hanks,

\footnotetext{
${ }^{3}$ C'est ici qu'interviendrait à mon sens la «mémoire discursive » ou le modèle mental du discours : à savoir, une restructuration hiérarchisée de l'information issue de la mise en discours.

${ }^{4}$ Voir aussi Ariel (2008, p. 2), Langacker (1996, p. 334) et Widdowson (2004, p. 8).
} 
1992) afin de profiler une figure (un nouveau référent ou une nouvelle conception d'un référent existant dans la mémoire discursive).

La deixis constitue une manière d'ancrer le discours à construire via la production d'un texte dans un contexte approprié (cf. Langacker, 2002a) : elle crée elle-même un nouveau contexte en fixant les valeurs particulières des paramètres contextuels de base (l'espace déictique - le moment déictique - les rôles de locuteur actuel et d'allocutaire actuel dévolus à tel ou tel participant du discours - la source du point de vue à l'œuvre) pour l'événement communicatif; une référence canoniquement déictique introduit la perspective subjective ou la source du point de vue à partir duquel ce discours est construit (voir aussi Oakley, 2009, p. 173-4, Rubba, 1996). Nous avons affaire à la deixis à chaque fois que nous devons avoir recours (par défaut) au contexte d'énonciation afin d'identifier le référent visé par le locuteur. L'emploi de la procédure référentielle déictique implique toujours une rupture, fût-elle brève, dans la continuité du discours jusqu'au moment de son occurrence.

Quant à l'anaphore, l'occurrence d'un anaphorique conjointement avec la proposition dans laquelle il apparait dans son ensemble, constitue un signal ou une instruction de maintenir le foyer d'attention déjà établi (ou présumé déjà établi) au moment de la parole - ainsi, l'état du modèle de discours tel qu'il existe au moment de l'emploi de l'anaphorique : l'anaphore signale qu'il ne doit pas y avoir de changement à cet égard, donc que ce serait "Business as usual», en somme - au sens: "pas d'introduction d'un nouveau référent, ou d'un nouveau foyer d'attention ». De cette façon, les référents d'anaphoriques peu ou non accentués et à hauteur basse (et qui sont de ce fait peu proéminents phonologiquement) sont supposés jouir d'un degré de foyer d'attention relativement élevé au moment de l'emploi. L'anaphore consiste donc en la reprise dans le cadre d'un fond contextuel donné (la représentation d'une situation quelconque) d'une figure (un objet de discours central au sein de ce fond) conjointement avec son fond contextuel. La prédication anaphorique dans son ensemble sert à étendre ce fond (voir aussi Kleiber, 1994, ch. 3).

Dans les deux cas, c'est la conceptualisation ou la représentation mentale, psychologique des référents qui est en jeu, que ces référents aient été rendus accessibles initialement de par la situation externe ou de par le co-texte précédent (voire suivant), ou bien par inférence (cf. Ariel, 1998). Il existe différents « champs » ou domaines de référence ${ }^{5}$ sur lesquels les procédures déictique tout comme anaphorique peuvent opérer : la situation d'énonciation (deixis pure, anaphore « exophorique »); le co-texte (deixis textuelle, anaphore métatextuelle); le discours environnant (deixis de discours, anaphore résomptive); la mémoire à long terme («anadeixis de reconnaissance/de rappel », anaphore résomptive); et l'évocation d'un monde fictif ou représenté (la Deixis am Phantasma de Bühler, 1934/2009).

La deixis n'est donc pas limitée, contrairement à ce que prétendait Bühler, à la référence «externe », à des objets disponibles de par la situation d'énonciation; et de même, l'anaphore n'est pas confinée à la reprise de référents ayant été introduits textuellement dans le co-texte antérieur (« anaphore » au sens strict) ou bien ultérieur (la « cataphore »). Voir Cornish (1999; 2010) pour des arguments dans ce sens.

${ }^{5}$ Voir à cet égard la notion bühlérienne de Zeigfelder («champs de pointage/indexicaux ») (Bühler, 1934/2009). 
Mais la relation entre deixis et anaphore est « orientée » (à la fois en termes de phylogenèse ${ }^{6}$ et d'ontogenèse ${ }^{7}$ ); ce ne sont pas des procédures indexicales « absolues 》 ou autonomes l'une par rapport à l'autre: comme l'a bien argumenté Lyons (1975) (cf. aussi Bühler, 1934/2009, Gerner, 2009 et d'autres), l'anaphore est dérivée de la deixis et en dépend, la deixis étant une procédure référentielle plus fondamentale. La plupart des marqueurs à même de réaliser l'anaphore peuvent également remplir une fonction déictique (ou sont dérivés de ceux qui sont spécialisés dans cette fonction). La véritable relation entre ces deux procédures indexicales se présente sous forme d'un continuum, avec un moyen terme : ce niveau intermédiaire, hybride a été dénommé «anadeixis » par Ehlich (1982) (voir aussi la sous-section 2.2 infra, qui présente mon interprétation de cette notion). La Figure 1 range 10 grandes catégories de marqueurs indexicaux sous forme d'une échelle de leurs propriétés indexicales intrinsèques ${ }^{8}$.

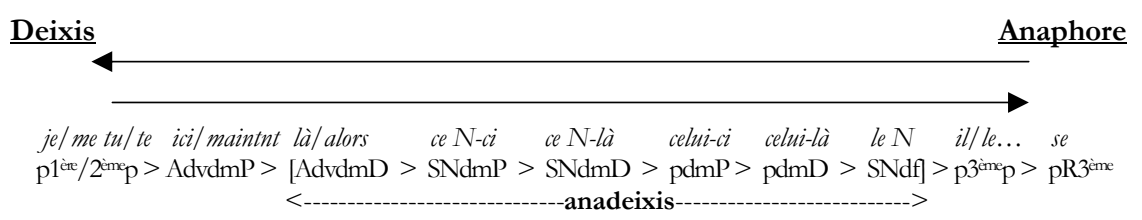

Figure 1 : Echelle de phoricité codée par certaines catégories d'expressions indexicales

La raison d'être de l'Echelle présentée dans la Figure 1 réside dans le degré d'indexicalité inhérente à chaque catégorie indexicale individuelle retenue. Les deux pôles sont occupés, respectivement, par les pronoms personnels de $1^{\text {ère }}$ et de $2^{\text {ème }}$ personne, qui sont intrinsèquement déictiques (fonctionnant de manière «tokenreflexive ») et ne peuvent s'employer anaphoriquement ; puis par les pronoms réfléchis clitiques de $3^{\text {ème }}$ personne, qui ne fonctionnent qu'anaphoriquement en termes de liage, et de plus à l'intérieur d'une proposition (leur champ d'opération est de ce fait fortement contraint). Ils relèvent donc de la « micro- » et non de la « macro-syntaxe ». Ce sont ces deux propriétés "polaires», diamétralement opposées, qui font que ces deux types de marqueurs fixent les limites supérieure et inférieure de l’Echelle. Dans

\footnotetext{
${ }^{6}$ C'est-à-dire, l'évolution de nouveaux types de marqueurs (ici, indexicaux) dans les langues du monde. Les expressions démonstratives semblent à cet égard tout à fait basiques, existant depuis la nuit des temps dans les langues actuelles et anciennes. C'est à partir de ces types d'expression que les autres formes indexicales ont été dérivées (par ex., dans les langues qui les ont, les articles définis, les pronoms de $3^{\text {ème }}$ personne).

${ }^{7}$ Autrement dit, l'évolution de la maitrise de sa langue maternelle par l'enfant. Celui-ci commence par utiliser les expressions démonstratives pour référer en situation (" (je) veux ça»; «donne-moi ça!» etc.) puis développe d'autres expressions indexicales plus tard dans l'acquisition de sa langue maternelle. Voir Clark (1978) à cet égard.

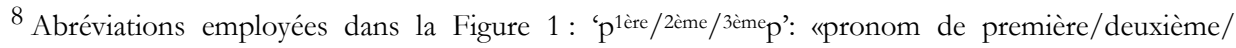
troisième personne»; 'P': « proximal »; 'D': « distal »; 'dm': « démonstratif»; 'adv': «adverbe »; 'SN': « syntagme nominal »; 'p': « pronom »; 'df': « défini »; 'R': « réfléchi ».
} 
les deux cas, une occurrence de chacun de ces types de marqueur détermine sa référence de façon quasi-automatique.

Les types d'expression à base démonstrative rangés entre ces deux catégories polaires sont ordonnés en fonction de la distinction proximale (marquée) vs. distale (non marquée) qu'ils peuvent porter morphologiquement (pronom dém./dét. dém. + nom $+-c i$ vs. pronom dém./dét. dém. + nom $+-l a ̈)-$ la variante marquée ayant un degré plus élevé de déicticité que la variante non marquée (cf. Langacker, 2002b, p. 34, Lyons, 1975). Les adverbes démonstratifs (par ex. maintenant/alors, ici/la $a^{9}$ ) sont placés à une position plus élevée que les SN lexicaux, et les $\mathrm{SN}$ lexicaux à une position plus haute que les pronoms correspondants. Toutes les catégories à base démonstrative sont placées au-dessus de la catégorie des SN définis sur cette Echelle : j’ai situé les SN définis à la limite inférieure de l'empan « anadéictique » dans la Figure 1, car même s'ils ne réalisent pas toujours une fonction indexicale ${ }^{10}$, ils peuvent néanmoins connaitre des emplois déictiques (voir le SN défini le président contenu dans le SP du président de l'exemple (6) (ligne 12) plus bas ${ }^{11}$ ), anadéictiques (voir le SN défini étendu le garçon quill y avait chez vous de l'exemple (5), lignes 7-8, également plus bas) ou anaphoriques. Leur degré intrinsèque de déicticité est donc plus bas que celui des catégories à base démonstrative retenues (voir aussi la caractérisation de Langacker, 2002b, p. 33), mais plus élevé que celui des pronoms de 3 ème personne inaccentués - dont l'emploi est normalement restreint à la seule fonction anaphorique.

Considérons plus en détail à présent la notion d'« anadeixis » en tant que telle.

\subsection{L'anadeixis}

L'«anadeixis » correspond au type de référence indexicale qui combine les procédures anaphorique et déictique à des degrés différents. Autrement dit, les expressions indexicales qui la réalisent sont anaphoriques dans la mesure où leur référent est déjà (potentiellement du moins) présent dans la représentation discursive supposée partagée par locuteur et allocutaire au moment de son emploi ; et ce référent est récupéré par ce biais. Cependant, ce référent est moins que hautement saillant au moment du renvoi, à la différence de la situation qui prévaut avec l'anaphore canonique (il est relativement peu saillant par rapport à d'autres référents qui viennent d'être évoqués). En même temps, une référence anadéictique est déictique dans la mesure où le locuteur a recours au contexte d'énonciation afin de réorienter l'attention de son allocutaire vers un référent qui, bien que potentiellement disponible au sein du contexte discursif au moment de l'énonciation, n'est pas celui auquel on s'attendrait pour une référence ultérieure à ce moment-là du discours.

Mais elle n'est pas canoniquement déictique, au sens où il n'y a aucun référent totalement nouveau qui est ainsi introduit dans le discours, et où tous les paramètres énonciatifs ne sont pas modifiés suite à cette référence.

\footnotetext{
${ }^{9}$ Voir de Mulder et Vetters (2008) pour l'adverbe temporel maintenant, et Kleiber (2008) sur ici et là.

${ }^{10}$ En effet, ils peuvent référer indépendamment en fonction de leur contenu lexical, lorsque celui-ci suffit à identifier leur référent de manière non-univoque.

11 Il s'agit ici d'une deixis «variable », en fait, selon l'occurrence d'emploi de ce SN par le client concerné.
} 
Il y a selon moi trois sous-types essentiels d'« anadeixis » : l'anadeixis 'stricte', l'anadeixis de reconnaissance/de rappel et la deixis de discours. L'anadeixis 'stricte' est réalisée par ce que Diessel (1999) ainsi que Kleiber (1990) appellent « les démonstratifs anaphoriques ».

\subsubsection{L'anadeixis 'stricte'}

Le cas prototypique d'anadeixis (ce que j'appelle «l'anadeixis 'stricte'») consiste en la reprise par des démonstratifs (pronoms et $\mathrm{SN}$ ), et parfois même par des $\mathrm{SN}$ définis dans certaines circonstances, d'un référent déjà évoqué dans le discours environnant, mais qui n'est que peu ou pas topical au moment où la récupération du référent est à faire; ou bien là où il y a "compétition » entre plus d'un référent d'un même type donné (voir l'exemple (1) plus bas); ou enfin où un référent de discours macro-topical vient d'être introduit, et doit être pleinement installé dans l'empan de mémoire à court terme de l'allocutaire. Dans aucun de ces cas de figure un pronom de $3^{\text {ème }}$ personne inaccentué ne serait à même d'assurer avec naturel le maintien pur et simple du référent visé - sa vocation indexicale étant canoniquement anaphorique (voir sa position «basse » dans l'Echelle de la Figure 1). En voici une illustration textuelle - un résumé de film comportant l'évocation de plusieurs individus et de leurs relations mutuelles :

(1) «Paris s'éveille (20h.40 Arte)

Âgé de 19 ans, Adrien (Thomas Langmann), $\theta$ mêlé à un vol d'argent, s'enfuit de Bordeaux où $i l$ vit avec $s a$ mère, et $\theta$ arrive à Paris chez son père, Clément (Jean-Pierre Léaud), qu'iln'a pas vu depuis quatre ans. Ce père est un être immature, qui se raccroche à $s a$ jeunesse perdue en ayant une maittresse de 19 ans, Louise

5. (Judith Godrèche). Elle rêve de devenir comédienne ou, tout au moins, speakerine à la télévision. Clément ne perd pas une occasion de la rabaisser. Pour tenir, elle se drogue (...)». (Extrait d'un résumé de film, Le Monde Supplément Radio-Télé, 29-30/04/07, p. 7).

Ici, le SN démonstratif ce père à la ligne 3 fonctionne "anadéictiquement», en pointant un référent qui vient d'être introduit mais qui n'a pas encore été rendu topical (c'est 'Adrien', le fils de Clément, qui est le macro-topique au moment où cette référence apparait). De toute façon, un renvoi plus conséquent que par le biais d'un simple pronom de $3^{\text {ème }}$ personne (masculin ici) aurait été nécessaire dans ce contexte, pour distinguer les deux référents mâles à l'œuvre.

Justement, à cet égard, il y a une légère gêne ressentie par le lecteur au moment de rencontrer le pronom féminin sujet elle à la $5^{\text {ème }}$ ligne (Elle rêve de devenir comédienne...). Car, tout comme le personnage 'Clément' au moment de la première reprise, et même si c'est le seul personnage féminin introduit à cet endroit du texte ('Louise', la maitresse de Clément), ce référent n'est pas encore topical: après tout, il vient tout juste d'être introduit dans le discours (voir aussi Ariel 1998, p. 212 à ce sujet). Un pronom démonstratif comme celle-ci ou un $\mathrm{SN}$ démonstratif elliptique comme cette dernière aurait été plus naturel comme marqueur indexical ici. Ce père à la ligne 3 et le démonstratif attendu celle-ci ou cette dernière à la ligne 5 auraient donc un fonctionnement anadéictique «strict» dans ce texte. 


\subsubsection{L'anadeixis de reconnaissance/de rappel}

La dimension déictique de la référence "anadéictique de reconnaissance » au moyen d'un marqueur indexical relève du fait que le locuteur dans ce type d'emploi oriente l'attention de son allocutaire vers une représentation conceptuelle présumée partagée, située dans sa mémoire à long terme (qu'elle soit épisodique ou sémantique); mais en même temps il y a une dimension «anaphorique » à de telles références, puisque ces emplois présupposent l'existence préalable de cette représentation partagée dans la mémoire de l'allocutaire. Il ne s'agit là en aucune façon d'une tentative de construire une telle représentation mémorielle - le propre d'une référence canoniquement déictique. Mais la composante "déictique» de cette référence indexicale hybride l'emporte néanmoins sur sa dimension anaphorique - le propre des références anadéictiques de reconnaissance. En voici un exemple :

(2) [Photo en noir et blanc d'un cercueil drapé d'un linceul, sur lequel est posée une rose (Publicité pour les Pompes Funèbres Générales, Le Monde 05/11/93) :]

Légende : « Notre métier est de vous aider dans ces moments-là »

Ici, le SN démonstratif ces moments-là a un caractère clairement « distal » (d'éloignement), les marques démonstratives ces...-là contrastant avec ces...-ci. Ce sont normalement des SN démonstratifs distaux, comme ces moments-là dans (2), qui réalisent cette fonction ; mais des SN définis étendus (par exemple, par une relative d'identification) peuvent l'exprimer également : voir le SN défini le garģon qu'ily avait chez vous dans l'exemple (5), lignes 7-8 (section 3).

Le cadre de référence dans lequel s'inscrit l'interprétation du SN démonstratif distal dans (2) est fourni par la «mise en scène» représentée dans la photo dont l'énoncé cité constitue la légende. Le caractère artificiel et peu réaliste de cette scène est censé symboliser un événement rituel (donc conçu génériquement), modalité qui est maintenue par la prédication dont fait partie l'expression en question (le nombre - pluriel - que celle-ci exprime est d'ailleurs conforme à cette modalité). La forme démonstrative du marqueur indexical (ces...-lä) ici, forme qui correspond à l'évidence à un haut niveau de déicticité, signale avec l'élément prédicatif de l'expression (le nom tête au pluriel moments) que son référent doit être retiré de la mémoire encyclopédique - de la mémoire à long terme, donc -, la situation évoquée ainsi étant considérée par l'auteur du message comme constituant une représentation mémorielle partagée, car stéréotypique. De ce fait, ce référent est rendu saillant. Il faut noter ici que l'entrée encyclopédique en question («les obsèques d'un être cher») est déjà évoquée par le contenu de la photo qui sert de cadre au texte, ainsi que par l'indication de la source du message lui-même (les Pompes Funèbres Générales). Cet exemple combine de ce fait référence déictique (pointage vers la représentation mémorielle partagée stéréotypique), et référence anaphorique : à ce titre, le texte de la légende fournit un commentaire sur cette situation, supposée à présent être familière au lecteur à travers la photo (c'est là la valeur que contribue à la référence du $\mathrm{SN}$ dans son ensemble la particule démonstrative suffixale $-l_{a}^{12}$ ).

\footnotetext{
12 Voir Kleiber (2008) pour des arguments selon lesquels là est un marqueur à vocation plutôt anaphorique que déictique.
} 
Francis Cornish

\subsubsection{La deixis de discours}

Avec la deixis de discours, c'est sur le discours environnant qui vient d'être construit qu'opère l'allocutaire afin de s'approprier (ou plutôt de créer, car c'est de cela qu'il s'agit ici) le référent visé. Le référent créé et rendu saillant par cette opération ne pré-existe pas en tant que tel à la référence déictique de discours. Ce n'est donc pas une reprise anaphorique - n'en déplaise à Piwek et alii $(2008$, p. 697) ou, de façon plus nuancée, à Kleiber $(2007, \$ 1)$ : pour les premiers, la deixis de discours est en réalité une forme d'anaphore de discours, arguant du fait que son mode d'interprétation exige d'avoir recours à l'amont ou à l'aval du discours environnant.

La deixis de discours ne peut en principe être réalisée que via des expressions démonstratives : pronoms ou $\mathrm{SN}$ à déterminant démonstratif. Les $\mathrm{SN}$ définis, eux, ne sont, en principe, pas à même de la réaliser. La raison essentielle tient à mon avis au fait que la composante lexicale dans ces derniers (nom tête plus ses dépendants éventuels) dénote une propriété qui est par défaut présupposée du référent visé (bien que, comme le montre Béguelin 1998, les SN définis puissent dans certaines circonstances servir à introduire des informations discursivement inédites); elle n'est de ce fait pas disponible, en principe, comme c'est le cas de la partie descriptive des SN démonstratifs, pour prédiquer une propriété nouvelle ou pour (re-)classifier le référent concerné. De plus, avec les démonstratifs, c'est à la fois la plus grande «force » référentielle qui caractérise les marqueurs démonstratifs (voir l'Echelle de phoricité dans la Figure 1), et le caractère non-présuppositionnel de leur composante descriptive, s'agissant de $\mathrm{SN}$ et non de simples pronoms, qui permettent à ce type de marqueur indexical de réaliser la deixis de discours. En outre, alors que le $\mathrm{N}$ des $\mathrm{SN}$ définis en tant que marqueurs indexicaux correspondra souvent à un nom hypéronyme de celui de l'antécédent textuel (là où celui-ci existe), celui des SN démonstratifs pourra relever d'une appréciation subjective du référent concerné de la part de l'énonciateur ${ }^{13}$. Ceci est en rapport direct avec la subjectivité qui caractérise les références déictiques par opposition aux renvois anaphoriques. Voir entre autres Lyons (1977), Guillot (2007), Himmelmann (1996) et Diessel (1999) sur la deixis de discours.

Tout comme dans le cas des références déictiques canoniques (situationnelles), avec la deixis de discours, le référent est livré en partant du fait même de l'occurrence du marqueur indexical concerné, en fonction du contexte d'énonciation particulier de cette occurrence. Le nom commun qui fonctionne comme tête du SN démonstratif dans (3) joue un rôle essentiel dans cette opération, car il sert à classifier le référent à construire par ce biais; et ce qui est prédiqué plus explicitement de ce référent fournit une orientation supplémentaire dans ce processus de construction. Je renvoie à

\footnotetext{
13 Voir Schnedecker (2006) pour les différents types de prédicat employés dans les SN démonstratifs en français, ainsi que Apothéloz (1995), Apothéloz et Chanet (1997) et [Reichler-]Béguelin (1995) pour de nombreux exemples attestés et analysés. Langacker (2002a, p. 24) met également l'accent sur la nécessaire subjectivité des marqueurs déictiques, dans la mesure où leur fonctionnement est inéluctablement ramené aux participants du discours (énonciateur et allocutaire) dans l'acte d'énonciation. Ces participants sont par défaut les « voyeurs » ou «percepteurs » (cf. Kleiber, 2007, \2) - anglais « viewers » - qui impriment leur point de vue sur le message. Voir Apothéloz et Chanet (1997) pour quelques-unes des propriétés des SN définis et démonstratifs comportant un nom tête nominalisé en emploi discursif.
} 
Cornish (1999, ch. 3) et (2010, p. 229-233) pour le rôle crucial rempli par la prédication indexicale dans le fonctionnement de l'anaphore, de la deixis et de l'anadeixis. L'exemple (3) illustre donc le fonctionnement de ce sous-type de procédure anadéictique.

(3) « Breaking the Waves, Arte 22h.25 FILM

(...) Le film commence lorsqu'elle [Bess, interprétée par Emily Watson] demande aux «sages » la permission d'épouser Jan, un homme qui n'est pas du village. Cette transgression est la première d'une longue liste à venir qui va crescendo, faisant de cette folle naïve une allégorie du Christ...» (Le Monde $10-11 / 10 / 10$, p. 2).

Tout comme le second SN démonstratif ici (cette folle naïve, ligne 4), le SN cette transgression est à l'évidence une caractérisation subjective en partie prise en charge par l'auteur de ce résumé de film (sans doute avec une touche d'ironie), et en partie imputée aux « sages » à qui Bess avait demandé l'autorisation d'épouser Jan (suivant les connotations religieuses du nom transgression) - le co-texte précédent constituant une description plutôt objective de l'intrigue du film. Il s'agit ici d'une condensation réifiante du contenu prédicatif de la phrase qui précède, impliquant une inférence selon laquelle épouser une personne qui ne vient pas de son village dans la situation évoquée constituerait une «transgression » (aux yeux des «sages » du village de Bess); par ce biais, ce $\mathrm{SN}$ fonctionne comme une instruction de créer un référent de discours ayant un niveau de saillance psychologique élevé. En fait, il est clair que ce SN démonstratif à caractère "appréciatif » signale le début d'un segment de discours qui sort des circonstances particulières de l'action du film (le résumé proprement dit), pour en faire une appréciation personnelle (le «sens» du film correspond selon l'auteur à une «allégorie du Christ» centrée sur son héroïne) ${ }^{14}$. Dans (3), c'est donc l'auteur, en prenant le parti des «sages » du village de Bess, qui prend clairement en charge cette unité du discours; il ne s'efface plus devant la description objective des faits. L'emploi du SN démonstratif à valeur de 'deixis de discours' marque donc le début d'une nouvelle unité de ce discours. A noter qu'un SN défini équivalent (\#La transgression...) n'aurait pas été aussi naturel, ni même acceptable en tant que tel, à ce moment du discours ${ }^{15}$.

La Figure 2 présente les différentes procédures de référence indexicale qu'on a vues jusqu'ici, également sous la forme d'une échelle :

Deixis pure > Deixis de discours $>$ Deixis de « reconnaissance » > Anadeixis 'stricte' > Anaphore pure $<-$

Figure 2 : Echelle des procédures de référence indexicales

\footnotetext{
14 Voir Cornish (2011) pour des exemples textuels anglais, où des SN démonstratifs à fonction déictique de discours servent à signaler des transitions entre deux unités de discours.

15 Voir aussi Apothéloz (1995) pour bon nombre d'exemples analogues comportant des marqueurs démonstratifs (voir en particulier ses exemples (40)-(43), p. 165), que l'auteur analyse de façon très semblable ; cependant, il persiste à appeler ceux-ci des « anaphoriques » (par ex. ibid. p. 165), ce qu'ils ne sont manifestement pas, selon la conception présentée ci-dessus).
} 
Dans cette Echelle, l'empan d'« anadeixis » s'étend entre la « deixis de discours » à gauche, et l'«anadeixis stricte» à droite. J'ai placé l'anadeixis «de reconnaissance» entre la «deixis de discours » et l'« anadeixis stricte », puisqu'il s'agit d'une procédure référentielle plus déictiquement orientée que cette dernière (car le référent ainsi visé n'est pas aisément accessible à l'allocutaire/au lecteur, mais a besoin d'être récupéré depuis la mémoire à long terme). Cependant, à la différence de la « deixis de discours », il existe bien un référent quelque part en aval de la référence ainsi effectuée : il est simplement moins immédiatement accessible que dans le cas de l'« anadeixis stricte $»^{16}$. Cette conception 'scalaire' montre clairement que, avec l'anadeixis stricte, l'aspect anaphorique sera dominant (l'anadeixis 'stricte' étant placée sur l'Echelle plus près du pôle « anaphore »), alors qu'avec la deixis de discours, c'est l’aspect déictique qui prédomine.

\section{Anaphoriques et « anadéictiques " comme diagnostics du discours}

Le discours, qui, comme nous l'avons vu dans la section 1 supra (revoir la définition de cette dimension dans le tableau 1), est toujours plus prégnant que le seul texte ou le contexte, sera particulièrement mis en évidence par le fonctionnement de certains marqueurs indexicaux, qu'ils soient déictiques, «anadéictiques » ou anaphoriques, au sein des textes. Voici quatre exemples d'extraits d'enregistrements de conversations spontanées ou bien d'entretiens informels (provenant tous du corpus PFC). A la différence des exemples écrits présentés jusqu'ici, les quatre extraits oraux que nous allons analyser dans cette section impliquent des interactions.

(4) Conversation libre $75 \times A B 1$ (corpus PFC)

(...) AB1 : Fais voir, est-ce que tu as des problèmes de, de, des yeux, que tu voies.

SB1 : Ouais. Aujourd'hui j'ai appris que j'avais une petite myopie.

$\mathrm{E}:$ Ah là, là, tu vas acheter des lunettes.

5. SB1: Ah non, je lui ai dit non. Je lui ai dit que c'était pas possible (rires). AB1 : Tiens, elle va chez, elle va chez l'oculiste, elle s'assoit. $<$ SB1 : un ophtalmo.> Bof, ou ophtalmo, c'est pareil. (...)

AB1 : ...Donc, elle y allait. La bonne femme, elle fait son métier, elle lui dit qu'elle a des problèmes de, aux yeux. (...).

Les trois interlocutrices de l'exemple (4) se connaissent évidemment bien, car elles se tutoyent. Le contexte interactif est donc celui d'une relation intime entre les interlocutrices, le degré de connaissances mutuelles étant de ce fait élevé. Dans sa première intervention, SB1 déclare aux deux autres participants qu'elle a « appris » le jour même qu'elle souffrait d'une "petite myopie». Cela laisse donc clairement entendre qu'elle vient de consulter une ophtalmologue, source évidente de ce diagnostic spécialisé. C'est donc ce scénario qui est indirectement évoqué par ce biais. C'est là un premier cas de « décalage » entre 'discours' et 'texte', tels que je les ai définis dans la section 1 supra (voir le tableau 1).

\footnotetext{
16 Voir Kleiber (2007) sur « la deixis mémorielle » et ses rapports avec la deixis « empathique ».
} 
Les emplois par la locutrice SB1 à la ligne 4 de cet extrait du pronom clitique datif lui sembleraient provenir du fait qu'elle s'est complètement replongée dans la situation qu'elle raconte, narration qui est donc empreinte de subjectivité. Elle ne prend de ce fait pas la peine d'expliciter sa référence à l'ophtalmo, qui fait nécessairement partie du scénario "aller chez l'ophtalmo» (ce serait donc un référent relevant du «focus implicite » chez Sanford et Garrod, 1981). Par l'emploi du pronom clitique datif lui à la ligne 4, donc, SB1 fait le pari que son référent (l'ophtalmo à qui elle avait affaire dans cette histoire) fait déjà partie de la représentation mentale de cette scène chez ses interlocuteurs, ou peut en être aisément inféré, et peut de ce fait être ciblé par un simple pronom de $3^{\text {ème }}$ personne.

A partir de la ligne 5, un changement de contexte est imprimé par AB1, qui

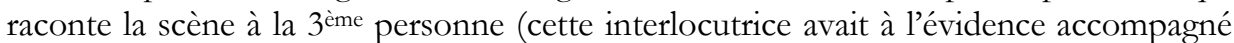
SB1 chez l'ophtalmologue). Elle reprend donc le récit à sa place, en employant à la ligne 5 un pronom de $3^{\text {ème }}$ personne féminin sujet renvoyant à $\mathrm{AB} 1$, elle-même présente dans la situation d'énonciation (ceci après une séquence, supprimée ici, sur la fonction respective des oculistes et des ophtalmologistes). Mais à présent, c'est un $\mathrm{SN}$ défini la bonne femme (ligne 8$)^{17}$ qui est employé pour renvoyer anaphoriquement à l'ophtalmologiste. Cette référence plus explicite de sa part peut être mise sur le compte du fait que AB1 a une vision plus « objective » ou du moins externe en tant que témoin de la scène dont il est question, que la protagoniste de l'affaire, $\mathrm{SB} 1^{18}$, et qu'elle la raconte en discours indirect (cependant, le SN défini comportant un «nom de qualité », la bonne femme, est assez péjoratif comme choix d'expression ici) ; et aussi pour éviter l'ambiguïté ou le hiatus qui serait créé(e) par l'emploi en succession rapide de deux pronoms sujets féminins à référence distincte ('SB1' d'une part, et 'l'ophtalmologiste', de l'autre).

(5) 42ayd1 Conversation libre (corpus PFC)

[La vie du locuteur à Saint-Germain Laval]

(...) Y : Tu as vu les, les enfants là de mes cousins. $<\mathrm{M}$ : Tu peux me rendre la tasse oui ?> (rires)

$\mathrm{Y}: \mathrm{Tu}$ as, tu as vu mes.

Y : On, on lavait gardé euh, d'ailleurs il est ingénieur maintenant.

5. Y : Il allait à, à Carnot.

E2 : Ah bon.

$\mathrm{Y}$ : Et je Pai eu en pension moi. <E2: Ah c'est le garcon qu'il y avait chez vous ?> Oui, oui. <E2 : Ah d'accord.> Oui, oui, oui.

$\mathrm{Y}$ : Je l'ai eu en pension pendant, au moins deux ans.

\footnotetext{
${ }^{17}$ Voir Corblin (2005) qui souligne le caractère exceptionnel dans les « chaînes de conversation » de ce type de marqueur à tête lexicale, mais quasi «pronominal» ici, pour renvoyer à des référents supposés connus par ailleurs des interlocuteurs. Corblin fait l'hypothèse que les interlocuteurs dans une conversation font partie d'une "communauté épistémique» restreinte, ce qui fait que les " chaînes référentielles » et "anaphoriques » composées (à l'oral) d'un nom propre repris par une suite de pronoms de $3^{\text {ème }}$ personne sont de mise, au détriment d'une variation de marqueurs de reprise (par exemple, entre des $\mathrm{SN}$ définis à tête lexicale variée), plus typique selon lui de l'écrit (type de discours «à interlocuteur générique »).

18 Qui, elle, comme on l'a vu, emploie de simples pronoms de $3^{\text {ème }}$ personne pour y renvoyer.
} 
Francis Cornish

Y : Qu'il venait coucher tous les soirs à la maison, il, il était à Carnot, il mangeait à Carnot, mais il venait coucher le soir. (...).

Dans l'exemple (5), le topique de cette séquence plutôt descriptive est 'les nouvelles des enfants des cousins de la locutrice Y' (topique évoqué à la première ligne de l'extrait). Ici nous constatons également l'emploi d'un simple pronom personnel de $3^{\text {ème }}$ personne pour reprendre un référent censé être connu de l'interlocutrice (voir les trois occurrences aux lignes 4 et 5), quoique non introduit explicitement ; cependant, cette fois il ne s'agit pas d'un référent faisant partie d'un scénario, et donc inférable par le biais des connaissances présumées de ce scénario (comme l'était celui des deux pronoms datifs à la ligne 5 de l'exemple (4)), mais d'un référent indépendant, censé être connu personnellement par ailleurs de l'interlocutrice (ici E2, l'enquêteuse : E2 vouvoie d'ailleurs $\mathrm{Y}$ comme on le voit à la ligne 8 ; cette forme du pronom peut être compris comme pluriel ici, mais E2 vouvoie Y par ailleurs dans la partie de la conversation précédant ce segment). Cependant E2 pourra bien avoir deviné, à partir du thème de ce segment de l'interaction ('les nouvelles des enfants des cousins de Y') annoncé à la ligne 1, que ce pronom masculin singulier renvoie à l'un des fils d'un cousin de $\mathrm{Y}$, mais sans savoir précisément lequel; pourtant, bien que E2 semble entériner cette référence présumée avec son « $A$ b bon» à la ligne 6 , de par son interruption aux lignes 7-8, on s'aperçoit que celle-ci avait néanmoins eu un doute sur l'identité exacte de cet individu (voir son interrogation aux lignes 7-8: "Ah c'est le garçon qu'il y avait chez vous? » comprenant un SN défini d'identification le garcon qu'il y avait chez, vous - SN qui est en fait un anadéictique de reconnaissance: notons la présence de la relative d'identification. La phrase n'est pas «attributive » mais « identificationnelle », et l'emploi d'un SN défini dans ce contexte présuppose bien la connaissance qu'a E2 de cet individu -voir la $\int$ 2.2.3 supra). La locutrice Y confirme cette interprétation immédiatement, E2 l'entérine, puis $\mathrm{Y}$ par la suite étoffe les caractéristiques identificatoires du référent pour E2, afin de bien confirmer, en la remettant à jour, sa représentation au sein de sa mémoire discursive (lignes 9-11) ${ }^{19}$.

Examinons à présent quelques SN définis employés en anaphore « indirecte ${ }^{20}$ (exemples (6) et (7) plus bas).

$$
\text { 75CAB1 Conversation libre (corpus PFC) }
$$

[Sur ce dont les gens discutent entre eux]

AB1 : (...) Il y a encore des gens qui vivent trop dans le passé en France mais c'est très, très, très fréquent en France, pas seulement euh, des gens

\footnotetext{
${ }^{19}$ Voir aussi l'accroc référentiel qui s'était produit suite à l'emploi d'un pronom à la place d'un marqueur plus explicite dans l'exemple (23) dans Johnsen (à paraître, \5.2).

${ }^{20}$ Une référence (anaphorique) «indirecte» est une référence réduite à un objet de discours censé être connu ou inférable par l'allocutaire ou le lecteur en l'absence d'une introduction co-textuelle explicite -ou même en sa présence : dans ce dernier cas de figure, ce référent est relié au référent explicitement évoqué en vertu d'une association quelconque. Voir Cornish (2005) pour une discussion plus ample.
} 
qui, qui restent dans leur petit rond, mais parce que les gens adorent parler famille, $<\mathrm{E} 1$ : Oui. $>$ et ils aiment bien l'histoire en général $<\mathrm{E} 1$ : Hum,

5. hum $>$ les gens, de toutes sortes hein, de toutes sortes. Au café, vous allez au café dans un village qu/ de quoi parlent-ils? De la politique, des voisins, et de la famille. Et la politique c'est le numéro un, hein, $<$ E1 : Oui, oui. $>$ oh oui, oh là ; ils parl/, ils parlent tous politique. Les chauffeurs de taxi, neuf fois sur dix, ils parlent politique, et bien, <E1 : Hum, hum.> et bien, <E1 : Hum,

10. hum. $>$ Il y a des pays où, il y a, il y a rien on monte dans le taxi, on paie la course et voilà, alors, le pauvre malheureux si on lui disait euh, 'alors que pensezvous du président?’ (XX) j’me serais affolée (rire). Non, peut-être pas craintif, mais n'ayant rien à dire, quoi. <E1 : Oui. $>$ Mais ça, ça a/. C’est peut-être un défaut mais, c'est encore comme ça malgré la télévision. $<\mathrm{E} 1$ : Oui.> Hum. Malgré la télévision. $<\mathrm{E}:$ Je pense que c'est une bonne chose les gens se parlent ils n'ont pas peur de.> Ah non ils n'ont pas peur de, oh non même ils, ils s'empoignent, ils se, <E1: Oui.> (XX) Ils se disent des choses incroyables et pu/, puis après ils sont pas fâchés quoi, non.... .

Le thème de l'extrait en (6) est les sujets de conversation des gens quand ils se rencontrent, surtout en France. Les contributions de l'enquêteuse se limitent pour l'essentiel à des phatiques, qui ponctuent le texte, montrant qu'elle est "présente » et qu'elle suit, en les approuvant, les propos de AB1. À partir de la ligne 8, il s'agit de chauffeurs de taxi en France qui « parlent surtout politique ».

Ensuite, la locutrice (AB1) compare la situation dans d'autres pays ( Il y a des pays où, il y a, il y a rien...» (lignes 9-10)). Ces constructions existentielles fonctionnent comme cadratifs, introduisant un nouveau sous-domaine de référence (étroitement dépendant de celui en vigueur précédemment). Comme auparavant, ce domaine de référence relève du stéréotype, les références à 'la course' (le trajet en taxi) et au chauffeur de taxi étant génériques. Notons l'emploi des SN et pronoms singuliers ici, évoquant la «classe» des chauffeurs de taxi (ainsi que des taxis) dans les pays étrangers. A remarquer, comme dans (7) plus bas, l'emploi du SN défini comportant un «nom de qualité », le (pauvre) malheureux (ligne 11), caractérisation hautement subjective exprimant (ici, ainsi que dans (7)) l'empathie de la locutrice pour ce type de chauffeur suite à la question (quelque peu indiscrète) de son client. Comme dans les exemples (4) et (5), cette reprise anaphorique est « indirecte », le référent en jeu n'ayant pas été explicitement introduit auparavant. Cependant, il peut être aisément inféré de par la situation stéréotypique évoquée (pour qu'il y ait 'trajet en taxi', il doit obligatoirement y avoir un 'chauffeur' dudit taxi) - encore un exemple du décalage entre 'texte' et 'discours' ici. A noter aussi que des SN démonstratifs (\#cette course et \#ce pauvre malheureux) n'auraient pas pu être employés à la place des SN définis respectifs ici : en effet, la généricité des références originales aurait disparu, seuls des renvois particuliers étant alors possibles (ce qui ne cadrerait pas du tout avec le contexte à l'œuvre, toutefois). 
Francis Cornish

(7) 75 cab1 Conversation guidée (corpus PFC)

[Sur les bombardements aériens pendant la seconde guerre mondiale en Bretagne]

...Mais euh, euh. Finalement euh quand euh nous avons été sous très gros bombardements, américains puis anglais et nous sommes (XX), après le bombardement anglais mon, mon père a dit 'non il $\mathrm{f} / \mathrm{il}$ faut partir il faut' tout le monde partait, lui il avait passé les quatre nuits, ou trois nuits, à, à

5. ramper dans les décombres pour aider les gens à mourir, parce qu'on pouvait pas sortir les gens de là c'était impossible. On s'est retrouvé sans eau, ni gaz, ni téléphone, ni électricité, et les pompiers, euh, euh c'était à $(\mathrm{XX})$, la ville est assez haute, <E1 : Hum, hum.> et la, la Loire est en bas. Et les malheureux allaient puiser de l'eau dans la Loire et remontaient pour arroser voyez c'était, c'était effarant... .

Dans l'exemple (7), on a affaire à une simple narration par la locutrice de la situation de sa famille pendant les bombardements alliés au cours de la 2ème Guerre Mondiale, l'enquêtrice E1 n'intervenant que par un seul phatique (ligne 8). Ici, rétroactivement, l'on comprend que 'les malheureux' en question (la référence à la ligne 8) sont 'les pompiers', introduits de façon ultra-rapide, presque «en passant», à la ligne 7, introduction suivie d'une préparation de l'interlocutrice à ce qui va être énoncé par la suite : à savoir, la grande différence de hauteur géographique relative entre la Loire en bas, où des quantités énormes d'eau étaient disponibles, et la ville en flammes dont il est question en haut.

Ce segment constitue en fait une sorte de "parenthèse » dans le récit, ce qui motiverait l'emploi d'un marqueur indexical plus conséquent qu'un simple pronom, rappelant ainsi le référent à l'œuvre. L'exemple pourrait être qualifié d'« hybride », dans le cadre de l'hypothèse des « insertions parenthétiques » proposée par Gachet (à paraitre), participant à la fois du "procédé 1 » (où le segment suivant l'interruption s'enchaîne directement sur celui qui la précède, car dans notre exemple la partie prédicative de la proposition hôte est directement appliquée aux 'pompiers' évoqués juste avant l'interruption); et en même temps du "procédé 2 », où le segment post-parenthétique s'enchaîne sur la parenthèse elle-même, dans la mesure où la conjonction et qui introduit la reprise du discours interrompu par la parenthèse peut être interprétée causalement : " et à cause de ça (" le fait que la ville est assez haute, et que la Loire est en bas ») », les «malheureux pompiers» se voyaient obligés de descendre au fleuve en bas puis remonter dans la ville en haut avec l'eau de ce fleuve pour y éteindre les incendies.

C'est précisément la nature de ce qui est prédiqué du référent du $\mathrm{SN}$ les malheureux ${ }^{21}$ qui permet d'associer par inférence 'les malheureux' dont il est question avec 'les pompiers de la ville bombardée' : c'est justement en cela qu'ils sont qualifiés de «malheureux» ici. Troisième cas donc de décalage ou de non-correspondance, entre texte et discours : celui-ci est à l'évidence donc bien plus riche et spécifique que ce que signifie le seul texte, en tant que tel.

${ }^{21}$ Le fait de «puiser de l'eau dans la Loire et de la remonter dans la ville haute pour arroser ». 
À la différence du SN défini singulier presque identique le pauvre malheureux de l'exemple (6), ici il serait parfaitement possible de remplacer ce SN par un SN démonstratif: ces malheureux, car la référence en (7) n'est pas générique, mais spécifique (quoique indéfinie, vague).

On voit donc ici que l'interlocutrice (et l'analyste) doivent faire de nombreuses inférences afin d'arriver à l'interprétation voulue. L'écart entre 'texte' et 'discours' est de ce fait considérable.

\section{Conclusions}

En guise de conclusion, voyons quelques implications des différents cas de référence indexicale qu'on a vus ici pour l'état de la mémoire discursive à l'œuvre c'est-à-dire, la représentation mentale structurée du discours alimenté par le texte ainsi que le contexte. La mémoire discursive est à la fois un construit théorique, postulé pour rendre compte des faits de discours (cf. la section 1), et un phénomène psychologiquement réel ${ }^{22}$.

Ces implications sont de deux ordres, essentiellement : 1) des modifications de la représentation discursive existant avant le renvoi indexical à l'objet de discours concerné; et 2) des changements de référent, et partant, l'installation d'une nouvelle représentation pour caractériser ces nouveaux référents au sein de la mémoire discursive.

Les renvois correspondant au premier cas de figure sont les suivants :

- Pour ce qui concerne l'anaphore «indirecte», il peut y avoir recatégorisation de la représentation du référent (ou «objet de discours») au sein de la mémoire discursive au cas où le genre et/ou le nombre du pronom de $3^{\text {ème }}$ personne diffère(nt) par rapport aux valeurs morpho-syntaxiques ${ }^{23}$

\footnotetext{
22 Elle s'inscrirait dans ce que les spécialistes appellent «la mémoire de travail » - cf. entre autres, Baddeley (1987), Cowan (1997) ; voir aussi Coulson (2001, ch. 4) sur l'implication du cerveau (rôles des hémisphères gauche et droit) dans la structuration du discours dans l'esprit des usagers, dans un cadre cognitiviste. Voir Cornish (1999, p. 209-228) pour une présentation synthétique des implications de la mémoire du travail pour la référence discursive indexicale.

${ }^{23}$ Voir comme exemple l'extrait du corpus PFC présenté par Johnsen (à paraitre : ex. (22)) :

(...) BL: Alors que c'est un peu bizarre enfin moi j'ai l'impression que si je connais une règle j'arrive quand même à l'appliquer.

E: M.

BL: Surtout que c'est q/ on, on leur demande pas non plus euh, tu vois euh incroyable quoi c'est, c'est plus euh, Tu vois l'accord du participe passé par exemple c'est vrai que c'est pas super évident mais, au bout de au bout de mille fois tu, tu la connais quoi (rire). (PFC, Liège, blaat1, LG).

Ici, il est évident que c'est le $\mathrm{SN}$ indéfini une règle dans la 1 ère ligne de la première intervention de $\mathrm{BL}$ qui constitue le «déclencheur d'antécédent ». Voir aussi les exemples et la discussion dans Cornish (1987, p. 249-257) pour la re-catégorisation que peuvent effectuer les pronoms marqués pour le genre et le nombre.
} 
Francis Cornish

portées par le déclencheur d'antécédent ${ }^{24}$, ou quand la partie descriptive $\mathrm{du}$ marqueur lexical apporte une (re-)classification ou prédique une propriété nouvelle du référent visé. Dans la conception développée ici, il y aura toujours un "déclencheur d'antécédent», même en l'absence d'un antécédent textuel classique. Dans les deux cas de figure mentionnés à l'instant, il s'agira d'ajouter des prédications à la caractérisation initiale du référent au sein de la mémoire discursive, le cas échéant en remplaçant le prédicat spécifiant le type ontologique d'entité à l'œuvre dans cette représentation. Ce prédicat remplirait la fonction de tête sémantique de la représentation.

- D'autre part, l'emploi référentiel d'un pronom de $3^{\text {ème }}$ personne va supposer l'existence dans la mémoire discursive du destinataire de la représentation discursive saillante d'un référent -qu'elle y ait été installée par introduction textuelle explicite ou par inférence : voir pour ce deuxième cas de figure, les occurrences du pronom datif lui dans (4) (ligne 5) provenant du scénario « aller chez l'ophtalmo ».

Dans (5), l'emploi par Y des pronoms l' et il supposant de sa part la présence d'une représentation saillante du référent visé dans la mémoire de travail de son allocutaire, E2, n'était pas justifié : c'est précisément une expression comme le SN étendu le garçon qu'il y avait chez. [moi], en emploi anadéictique de reconnaissance, qui aurait été plus appropriée de la part de Y pour rappeler à la conscience de E2 le référent qu'elle avait à l'esprit à ce moment-là du discours. En fait, ce $\mathrm{SN}$ défini, avec le pronom de $2^{\text {ème }}$ personne vous régi par la préposition chez, était utilisé par E2 à la ligne 8 pour vérifier que c'était bien ce référent que ciblaient les pronoms masculins singuliers de $\mathrm{Y}$.

- Des $\mathrm{SN}$ définis à fonction purement anaphorique peuvent également cibler un référent supposé saillant dans la $\mathrm{MD}$, mais non explicitement introduit : voir les SN « de qualité » la bonne femme, ciblant l'ophtalmologiste qui avait reçu SB1 dans (4) (ligne 8), le pauvre malheureux dans (6) (ligne 11) et les malheureux dans (7) (ligne 8). Ces expressions nominales définies sont empreintes de subjectivité, la première suggérant une attitude péjorative, négative, la seconde et la troisième clairement une empathie et une compréhension de la situation dans laquelle se trouverait le référent visé. Il semble donc que le point de vue subjectif ainsi que sa source, imprimés sur le référent via le renvoi en question, doivent également figurer dans la représentation en évolution du référent de discours.

- Il faudra tenir compte à ce titre des $\mathrm{SN}$ et pronoms démonstratifs employés en anadeixis stricte (voir l'exemple (1)), qui ciblent un référent déjà représenté en $\mathrm{MD}$, mais en le (re-)topicalisant, l'amenant sur le devant de «la scène cognitive », autrement dit à la conscience actuelle du destinataire. Cette

\footnotetext{
${ }^{24}$ Un « déclencheur d'antécédent» peut correspondre à une occurrence d'énoncé, à l'objet d'une perception, ou à un geste sémiotiquement pertinent. Voir Cornish (2010) pour une mise au point récente de cette notion.
} 
fonction ne change pas le contenu de la représentation discursive concernée, mais elle en modifie le statut discursivo-cognitif existant au moment du renvoi. Ce phénomène implique que les représentations discursives des référents à l'œuvre doivent être marquées différentiellement pour le statut attentionnel qu'ils revêtent à chaque point du déroulement du discours dans le temps.

Dans les cas évoqués ci-dessus, le référent installé dans la MD n'est pas modifié en tant que tel - c'est le même référent qu'avant le renvoi, mais certains de ces renvois modifient la représentation discursive qui peut exister antérieurement. De toute façon, la représentation mentale du référent visé sera obligatoirement remise à jour de par l'ajout de la propriété ou relation qui en sera prédiquée dans la proposition hôte dans son ensemble.

Dans le second cas de figure, en revanche, le marqueur indexical conjointement avec la prédication hôte peut même effectuer un changement de référent, qui sera installé au moyen d'une toute nouvelle représentation discursive :

- Dans le cas de la référence déictique canonique, ainsi que de la deixis de discours, l'acte discursif consiste à introduire un tout nouveau référent (quoique construit à partir du résultat de la mise en discours qui vient d'être effectuée, dans le cas de la deixis de discours). Il s'agira donc de la création d'une nouvelle représentation discursive en $\mathrm{MD}$, le référent étant inédit. C'est le cas du référent ciblé par le SN démonstratif cette transgression dans (3). Ici, la représentation discursive devra comprendre l'indication que, aux yeux des « sages » du village de Bess au Danemark à l'époque, on n'épousait pas quelqu'un qui ne venait pas de ce même village. C'est précisément en cela qu'une telle demande d'autorisation de sa part pouvait constituer une « transgression », quasi religieuse, aux yeux des « sages » en question.

- Encore une fois, comme dans (4), (6) et (7), le point de vue, ainsi que sa source, imprimés par la référence en question devront être indiqués dans la représentation discursive à l'œuvre.

En somme, on voit par là que les différents types de marqueurs indexicaux à l'œuvre dans le discours ont une fonction discursive bien plus "pro-active» que celle qui consiste simplement à permettre à l'allocutaire ou au lecteur d'identifier l'objet de discours visé par le locuteur ou le scripteur.

\section{Remerciements}

Cet article est la version révisée d'une présentation (invitée) faite lors de l'Atelier de recherche en linguistique française «Entre syntaxe et discours » tenu du 11 au 12 novembre 2010 à l'Université de Neuchâtel. Je tiens à remercier les organisateurs de cet Atelier de m'y avoir invité, ainsi que les participants pour la discussion qui a suivi mon exposé. Je remercie également Laure Anne Johnsen, Laurie Buscail, Nathalie Rossi-Gensane et Georges Kleiber ainsi que deux relecteurs «internes » et un de la revue $L I N X$, pour leur relecture de versions antérieures de ce texte. 


\section{Références bibliographiques}

APOTHÉLOZ, D. (1995), «Nominalisations, référents clandestins et anaphores atypiques », TRANEL 23, p. $143-173$.

ApothÉLOZ, D. \& CHANET, C. (1996), «Défini et démonstratif dans les nominalisations », in De Mulder, W., Tasmowski-De-Ryck, L. \& Vetters, C. (eds.), Relations anaphoriques et (in)cohérence. Amsterdam, Rodopi, p. 159-186.

Apothéloz, D. \& [Reichler-]BÉGuelin, M-J. (1995), «Construction de la référence et stratégies de désignation », TRANEL 23, p. 227-271.

ARIEL, M. (1998), "The linguistic status of the 'here and now” ", Cognitive Linguistics 9 (3), p. 189-237.

ArIEL, M. (2008), Pragmatics and Grammar, Cambridge, Cambridge University Press.

Auer, P. (1992), «Introduction: John Gumperz’ approach to contextualization », in Auer, P. \& di Luzio, A. (eds.), The Contextualization of Language, Amsterdam, John Benjamins, p. 1-37.

Baddeley, A. (1987), Working Memory, Oxford, Clarendon Press, Oxford University Press.

BÉGUELIN, M-J. (1998), «Le statut des informations non identifiantes dans le SN défini anaphorique », in Forsgren, M., Jonasson, K. \& Kronning, H. (eds.), Prédication, assertion, information, Actes du colloque d’Uppsala en linguistique française. 6-9 juin 1996, Acta Universitatis Upsaliensis, p. 463-474.

Benveniste, E. (1974), Problèmes de Linguistique Générale Tome II, Paris, Gallimard.

Berrendonner, A. (1983), "Connecteurs pragmatiques et anaphore », Cabiers de Linguistique Française 5, p. 215-246.

BRISARD, F. (ed.) (2002), Grounding. The epistemic footing of deixis and reference, Berlin \& New York, Mouton de Gruyter.

BÜHLER, K. (2009)[1934], Théorie du langage. La fonction représentationnelle du langage, Traduction, notes et glossaire par Didier Samain, Agone, Marseille, Collection Banc d'Essais.

CLARK, E. (1978), «From gesture to word: On the natural history of deixis in language acquisition. », in Bruner, J. S. \& Garton, A. (eds.), Human Growth and Development. Oxford, Oxford University Press, p. 85-120.

Corblin, F. (2005), «Les chaînes de la conversation et les autres », in Gouvard, J-M. (ed.), De la langue au style, Lyon, Presses Universitaires de Lyon, p. 233-254.

CORNISH, F. (1987), « Anaphoric pronouns: Under linguistic control, or signalling particular discourse representations? ", Journal of Semantics 5, p. 233-260.

CORNISH, F. (1999), Anaphora, Discourse and Understanding. Evidence from English and French, Oxford, Clarendon Press.

CORNiSH, F. (2005), « Degrees of indirectness : two types of implicit referents and their retrieval via unaccented pronouns », in Branco, A., McEnery, T. \& Mitkov, R., (eds.) Anaphora Processing: Linguistic, cognitive and computational modelling, Amsterdam/Philadelphia, John Benjamins, Current Issues in Linguistic Theory 263, p. 199-220. 
CORNISH, F. (2009), «Text and discourse as context. Discourse anaphora and the FDG Contextual component", in Keizer, E. \& Wanders, G., (eds.), numéro thématique des Working Papers in Functional Discourse Grammar (WP-FDG-82): The London Papers I, p. 97-115. Disponible au site web http://home.hum.uva.nl/fdg/working_papers/WP-FDG-82_Cornish.pdf

CORNISH, F. (2010), « Anaphora: Text-based or discourse-dependent? Functionalist vs. formalist accounts », Functions of Language 17 (2), p. 207-241.

CORNISH, F. (2011), "'Strict' anadeixis, discourse deixis and text structuring », Language Sciences 33 (5), p. 753-767.

COUlson, S. (2001), Semantic Leaps: Frame-shifting and conceptual blending in meaning construction, Cambridge, Cambridge University Press.

Cowan, N. (1997), Attention and Memory: An integrated framework, Oxford, Clarendon Press, Oxford University Press.

De Mulder, W. \& VetTers, C. (2008), «Le sens fondamental de maintenant: un tokenreflexive », Cabiers Chronos 20, p. 15-33.

DiESSEL, H. (1999), Demonstratives. Form, function, and grammaticalization, Amsterdam/Philadelphia, John Benjamins.

DiEssel, H. (2006), «Demonstratives, joint attention, and the emergence of grammar », Cognitive Linguistics 17(4), p. 463-489.

EHLICH, K. (1982), «Anaphora and deixis: Same, similar, or different? », in Jarvella, R.J. \& Klein, W., (eds.), Speech, Place and Action. Studies in deixis and related topics, Chichester, Wiley, p. 315-338.

GACHET, F. (à par.), «Sorties de parenthèses », in Groupe de Fribourg (éd.), Grammaire de la période II. Les parenthèses, Berne, Peter Lang.

Gerner, M. (2009), «Deictic features of demonstratives: A typological survey with special reference to the Miao group ", Canadian Journal of Linguistics/Revue canadienne de linguistique 54(1), p. 43-90.

Guillot, C. (2007), «Entre anaphore et deixis : l'anaphore démonstrative à fonction résomptive ", in Trotter, D. (ed.), Actes du XXIVe Congrès International de Linguistique et de Philologie Romanes, Vol. 3, Aberystwyth 2004, Tübingen, Max Niemeyer Verlag, p. 307315.

GumperZ, J. J. (1992a), «Contextualization and understanding», Ch. 8 in Duranti, A. \& Goodwin, C. (eds.), Retbinking context: Language as an interactive phenomenon, Cambridge, Cambridge University Press, p. 229-252.

Gumperz, J. J. (1992b), «Contextualization revisited», in Auer, P. \& di Luzio, A. (eds.), The Contextualization of Language, Amsterdam, John Benjamins, p. 39-53.

HANKs, W. F. (1992), «The indexical ground of deictic reference», in Duranti, A. \& Goodwin, C. (eds.), Rethinking Context: Language as an interactive phenomenon, Cambridge, Cambridge University Press, p. 43-76.

HimmelmanN, N. P. (1996), «Demonstratives in narrative discourse: A taxonomy of universal uses », in Fox, B.A. (ed.), Studies in Anaphora. Amsterdam/Philadelphia, John Benjamins, p. 205-254. 
Francis Cornish

JARVELLA, R.J. (1979), «Immediate memory and discourse processing », in Bower, G.B. (ed.), The Psychology of Learning and Motivation Vol. 13, New York, Academic Press.

JOHNSEN, L. A. (à paraitre), «Anaphore pronominale indirecte en micro- et macro-syntaxe », in Barbazan, M. (ed.), Actes du colloque Énonciation et texte au coeur de la grammaire. Regards croisés: neuro/psycholinguistique, psychologie cognitive, linguistique, didactique, Université de Toulouse-Le Mirail, 11-13 mars 2009, Presses Universitaires de Rennes.

KLEIBER, G. (1990), «Sur l'anaphore démonstrative », in Charolles, M., Fisher, S. \& Jayez, J. (eds.), Le discours. Représentations et interprétations, Nancy, Presses Universitaires de Nancy, p. 243-263.

KLEIBER, G. ( 1994), Anaphores et pronoms, Louvain-la-Neuve, Duculot.

KLEIBER, G. (2007), «Des démonstratifs mémoriels aux démonstratifs de point de vue », in Begioni, L. \& Muller, C. (eds.), Problèmes de sémantique et de syntaxe. Hommage à André Rousseau, Villeneuve d'Ascq, Editions du Conseil Scientifique de l'Université Charles de GaulleLille 3, p. 373-392.

KLEIBER, G. (2008), « Comment fonctionne ICI? », Cabiers Chronos 20, p. 113-145.

LANGACKER, R. W. (1996), «Conceptual grouping and pronominal anaphora », in Fox, B. (ed.), Studies in Anaphora, Amsterdam, John Benjamins, p. 333-378.

LANGACKER, R.W. (2002a), « Deixis and subjectivity », in Brisard (ed.), p. 1-27.

LANGACKER, R.W. (2002b), "Remarks on the English grounding systems », in Brisard (ed.), p. 29-38.

LyONS, J. (1975), «Deixis as the source of reference », in Keenan, E.L. (ed.), Formal Semantics of Natural Language, Cambridge, Cambridge University Press, p. 61- 83.

LyOns, J. (1977), Ch. 15 : «Deixis, space and time», in Semantics, Vol 2. Cambridge, Cambridge University Press, p. 636-724.

OAKLeY, T. (2009), «Mental spaces », in Brisard, F., Östman, J-O. \& Verschueren, J. (eds.), Grammar, Meaning and Pragmatics, Amsterdam/Philadelphia, John Benjamins, p. 161-178.

PARIsI, D. \& CASTELFRANCHI, C. (1977), «The discourse as a hierarchy of goals », Signs of Change 1.2, p. 31-67.

Piwek, P., Beun, R-J. \& Cremers, A. (2008), « 'Proximal' and 'distal' in language and cognition: Evidence from deictic demonstratives in Dutch », Journal of Pragmatics 40, p. 694-718.

[REICHLER-]BÉGUELIN, M-J. (1988), « Anaphore, cataphore et mémoire discursive », Pratiques 57 , p. $15-43$.

[REICHLER-]BÉGUELIN, M-J. (1995), «Alternatives et décisions lexicales dans l'emploi des expressions démonstratives », Pratiques 85, p. 58-87.

RubBA, J. (1996), "Alternate grounds in the interpretation of deictic expressions », in Fauconnier, G. \& Sweetser, E. (eds.), Spaces, Worlds, and Grammar, Chicago, University of Chicago Press, p. 227-261.

SANFORD, A.J. \& GARROD, S.C. (1981), Understanding Written Language. Explorations in comprehension beyond the sentence, Chichester, Wiley \& Sons Ltd. 
Indexicaux, discours et mémoire discursive : ce que les premiers révèlent du second et de la troisième

SCHNEDECKER, C. (2006), «Les démonstratifs 'prédicatifs' : qu'est-ce qui limite leur apport informatif ? ", Langue Française 152, p. 39-55.

Verhagen, A. (2005), Constructions of Intersubjectivity. Discourse, syntax, and cognition, Oxford, Oxford University Press.

WidDowson, H.G. (2004), Text, Context, Pretext. Critical issues in discourse analysis, Oxford, Blackwell. 